\title{
Neratinib in Early-Stage Breast Cancer: A Profile of Its Use in the EU
}

\author{
Sohita Dhillon ${ }^{1}$
}

Published online: 3 January 2019

(c) Springer Nature 2019, corrected publication 2019

\begin{abstract}
Neratinib $\left(\right.$ Nerlynx ${ }^{\circledR}$ ) is an oral, irreversible pan-human epidermal growth factor receptor (HER) tyrosine kinase inhibitor of HER1, HER2 and HER4. Neratinib therapy for 12 months significantly reduced the risk of invasive disease recurrence or death relative to placebo at both 2 and 5 years post-randomization in the pivotal ExteNET trial in women with early-stage HER2-positive breast cancer who had completed adjuvant trastuzumab. Subgroup analyses showed that patients with hormone receptor (HRc)-positive disease derived greater benefit with neratinib than patients with HRc-negative disease, and patients who initiated neratinib within 1 year of completing trastuzumab had better outcomes than those who started treatment 1-2 years after trastuzumab. This led to the approval of neratinib in the EU as extended adjuvant therapy for patients with early-stage HRc-positive, HER2-positive breast cancer and who are less than 1 year from completion of prior adjuvant trastuzumab-based therapy. It is the first agent of its class to be approved in the EU in this setting. As with other tyrosine kinase inhibitors, diarrhoea, which was manageable with antidiarrhoeal prophylaxis and/or dose modifications, was the most common any-grade or grade $\geq 3$ treatment-emergent adverse event with neratinib. Thus, current evidence indicates that neratinib provides a valuable option to reduce the risk of recurrence in this setting and has been included in the updated ESMO patient guide as an extended adjuvant therapy for some patients.
\end{abstract}

\section{Adis evaluation of neratinib in early-stage} HER2-positive breast cancer

Oral, irreversible pan-HER tyrosine kinase inhibitor Improves invasive disease-free survival in patients who have completed adjuvant trastuzumab therapy

Patients with HRc-positive disease who receive neratinib within 1 year of completion of trastuzumab derive the greatet treatment benefit

Diarrhoea is the main toxicity, which is manageable with antidiarrhoeal prophylaxis and/or dose modifications

Additional information for this Adis Drug Q\&A can be found at https://doi.org/10.6084/m9.figshare.7418471.v1.

Sohita Dhillon

demail@springer.com

1 Springer, Private Bag 65901, Mairangi Bay, Auckland 0754, New Zealand

\section{What is the Rationale for Developing Neratinib?}

A better understanding of the complex molecular heterogeneity of breast cancer over the last several decades has led to the development of targeted therapies, such as the antihuman epidermal growth factor receptor 2 (HER2) monoclonal antibody trastuzumab, a mainstay in the treatment of patients with HER2-positive disease [1]. HER2 (ErbB2) is a member of the ErbB family of tyrosine kinase receptors, consisting of the epidermal growth factor receptor (EGFR, ErbB1, HER1), ErbB3 (HER3) and ErbB4 (HER4) [2]. ErbB receptor signalling plays a key role in the regulation of cell proliferation, survival, differentiation and migration [2]. Deregulation of signalling via these receptors (primarily HER1 and HER2) has been implicated in several types of cancers, including breast cancer [2]. HER2 is overexpressed in approximately $20-30 \%$ of breast cancer tumours and is associated with more aggressive disease, higher recurrence rates and increased mortality [1].

In early-stage HER2-positive breast cancer, standard adjuvant therapy with chemotherapy and trastuzumab for 1 year was associated with an absolute improvement of $6-12 \%$ in disease-free survival and 6-9\% in overall survival during 
longer-term follow-up (8-12 years); however, $15-20 \%$ of patients had disease recurrence [3]. Several options have been explored to escalate standard adjuvant therapy, including extending trastuzumab treatment to 2 years and combining trastuzumab with lapatinib (dual HER1 and HER2 inhibitor) or bevacizumab (anti-vascular endothelial growth factor monoclonal antibody) [3, 4]. However, no significant clinical benefit was demonstrated with these treatments [3]. The search for alternative therapies led to the development of the oral, irreversible, pan-HER tyrosine kinase inhibitor neratinib $\left(\right.$ Nerlynx ${ }^{\circledR}$ ) as an extended adjuvant treatment for patients with early-stage, HER2-positive breast cancer who have completed trastuzumab-based adjuvant therapy $[4,5]$. This review focuses on the clinical evidence for its use in the extended adjuvant setting, with a particular focus on the patient population for which it is approved in the EU, i.e. patients with hormone receptor (HRc)-positive, HER2positive tumours who initiate neratinib $<1$ year from completing trastuzumab-based adjuvant therapy [6]. Neratinib is the first anti-HER2 agent to be approved in the EU in this indication [7].

\section{How Does Neratinib Work?}

Neratinib binds irreversibly to a conserved cysteine residue in the ATP-binding pocket of HER1, HER2 and HER4, and reduces receptor autophosphorylation, resulting in sustained inhibition of downstream signal transduction events and cell cycle regulatory pathways, leading to cell cycle arrest and apoptosis [8]. Neratinib is highly selective for the ErbB family members and does not significantly inhibit several other kinases, including Akt, cyclin D1/cdk4, c-Raf and c-met [8]. In preclinical studies, neratinib inhibited the proliferation of HER1- and/or HER2-overexpressing cell lines, including breast cancer and epidermal carcinoma cell lines, and inhibited tumour growth in several HER1- and HER2-dependent xenograft models $[8,9]$.

By simultaneously inhibiting multiple members of the ErbB family, neratinib has the potential to overcome trastuzumab resistance mechanisms, such as compensatory crosstalk between receptors [10]. Indeed, neratinib was shown to be growth inhibitory in HER2-positive breast cancer cell lines with innate and acquired resistance to trastuzumab [9]. Furthermore, combination treatment with neratinib and trastuzumab had an additive effect in trastuzumab-sensitive cell lines and in cell lines with acquired resistance to trastuzumab, as indicated by significantly greater inhibition of proliferation with the combination than with each agent alone [9]. This improved activity of neratinib in combination with trastuzumab may be due to the different mechanisms of action of trastuzumab (binds to the extracellular domain of HER2 and induces ligand-dependent activation of HER receptors) and neratinib (binds to the intracellular ATP-binding site and inhibits receptor autophosphorylation) [11]. An additive tumour growth inhibitory effect of neratinib plus trastuzumab was also seen in a xenograft model of HER2-positive breast cancer sensitive to trastuzumab [9].

Oestrogen signalling plays a key role in breast carcinogenesis, and crosstalk between the oestrogen receptor (ER) and HER2 has been implicated in the development of resistance to endocrine- and HER2-targeted therapies [12]. The inhibition of HER2 signalling by targeted therapies is believed to activate/restore ER signalling, providing an escape mechanism for the tumour cells and resulting in resistance to HER2-targeted therapies [12, 13]. Endocrine therapy can also activate HER2 signalling and lead to resistance to HER2-directed therapy [12]. Thus, combining ERand HER2-targeted therapies to inhibit both signalling pathways simultaneously has the potential to improve clinical outcomes (Fig. 1). Indeed, a preclinical study showed that neratinib plus fulvestrant but not fulvestrant alone maintained complete tumour responses in mice with ER-positive, HER2-positive xenografts that had responded to trastuzumab plus paclitaxel [14]. In the clinical setting, the benefit of 12 months' extended adjuvant treatment with neratinib in the pivotal ExteNET trial in patients with early-stage HER2positive breast cancer was seen largely in the subgroup of patients with HRc-positive disease [13], the majority (93\%) of whom were receiving concomitant endocrine therapy [15] (Sect. 4).

\section{For Whom is Neratinib Indicated?}

In the EU, neratinib is indicated for the extended adjuvant treatment of adults with early-stage HRc-positive, HER2overexpressed/amplified breast cancer and who are $<1$ year from the completion of prior adjuvant trastuzumab-based therapy [6]. Table 1 provides a summary of the EU prescribing information for neratinib in this indication.

\section{What is the Clinical Efficacy of Neratinib?}

The large ( $n=2840)$, randomized, double-blind, multicentre, placebo-controlled, phase 3 ExteNET trial assessed the efficacy of oral neratinib as extended adjuvant therapy in patients with early-stage HER2-positive breast cancer who had completed adjuvant therapy with trastuzumab [15]. The trial included patients aged $\geq 18$ years $(\geq 20$ years in Japan) with locally (and subsequently centrally confirmed in $60 \%$ of primary tumour specimens) invasive HER2-positive breast cancer stage 1-3 (later amended to stage 2-3; Fig. 2) without evidence of recurrence. Patients had to have completed $\leq 2$ years' trastuzumab-based therapy (amended 


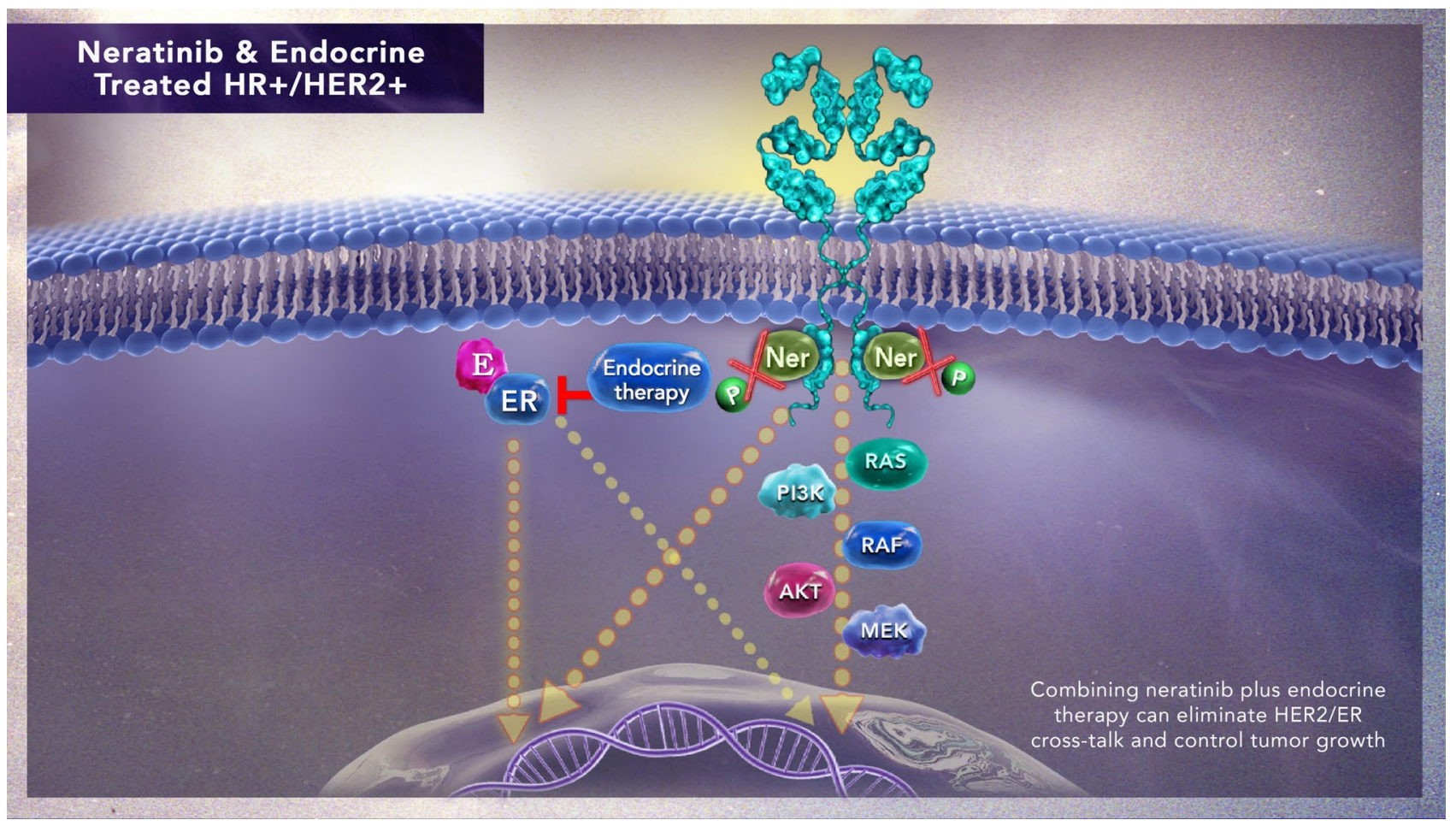

Fig. 1 Inhibition of ER/HER2 cross-talk with neratinib plus endocrine therapy. $E$ oestrogen, $E R$ oestrogen receptor, $H R$ hormone receptor, $H E R 2$ human epidermal growth factor receptor 2

later to 1 year; Fig. 2) before randomization, have an Eastern Cooperative Oncology Group (ECOG) performance status score of 0 or 1, normal organ function and a left ventricular ejection fraction (LVEF) within normal range [15]. HRc status (assessed locally) had to be known and it was recommended that patients receive concurrent adjuvant endocrine therapy for HRc-positive disease $[15,16]$.

Patients received neratinib ( $240 \mathrm{mg}$ once daily) or placebo for 12 months, unless there was disease recurrence, new breast cancer, an intolerable adverse event (AE) or withdrawal of consent. Randomization was stratified according to HRc status (positive or negative), nodal status $(0,1-3$ or $\geq 4$ ) and trastuzumab adjuvant regimen (given sequentially or concurrently with chemotherapy) [15]. The median time from the last trastuzumab dose to randomization was 4.4 and 4.6 months in the neratinib and placebo groups, respectively; 2297 (80.9\%) patients had completed trastuzumab therapy within 1 year of initiating study treatment. Demographic and disease characteristics at baseline were generally similar between the neratinib and placebo groups. At baseline, across the two groups, the median age of patients was 52 years; $57 \%$ had HRc-positive disease (i.e. ER- and/ or progesterone receptor-positive); 32, 40 and $9 \%$ of patients had stage T1, T2 and $\geq \mathrm{T} 3$ tumours, respectively; previous trastuzumab treatment was administered concurrently with chemotherapy in $62 \%$ and sequentially in $38 \%$ of patients; 24,47 and $30 \%$ of patients had 0,3 and $\geq 4$ positive nodes, respectively. In patients with HRc-positive disease, 93\% were receiving concomitant endocrine therapy [15].

The primary endpoint was invasive disease-free survival (DFS; see Table 2 for definition) assessed in the intent-totreat population [15]. Primary efficacy analyses were conducted at 2 years (cut-off date July 2014), a sensitivity analysis of efficacy outcomes was conducted at 5 years (cut-off date March 2017) and an analysis of OS will be undertaken after 248 events have occurred [15, 16]. As most patients had completed the trial at 2 years after randomization, reconsent from patients was required for extended follow-up; 2117 patients re-consented to retrospective collection of data between 2 and 5 years, and survival data beyond year 5 [15].

\subsection{Intent-to-Treat Population}

Neratinib treatment for 12 months significantly reduced the risk of invasive disease recurrence or death relative to placebo in women with early-stage HER2-positive breast cancer who had completed adjuvant trastuzumab therapy (Table 2) $[6,15]$. At 2 years (primary endpoint) $[6,15]$ and at the 5-year follow-up [16], neratinib recipients in the intent-to-treat population had significantly higher rates of invasive DFS than placebo recipients, corresponding to a 34 and $27 \%$ relative reduction in the risk of invasive disease recurrence or death at the two timepoints (Table 2). A separation between the Kaplan-Meier curves 
Table 1 Prescribing summary of neratinib $\left(\right.$ Nerlynx $\left.^{\circledR}\right)$ in the treatment of early-stage hormone receptor positive HER2-overexpressed/ amplified breast cancer in the EU [6]

How is neratinib available?

$40 \mathrm{mg}$ film-coated tablet

What is the administration regimen of neratinib?

$240 \mathrm{mg}$ (six $40 \mathrm{mg}$ tablets) taken orally once daily, with food and preferably in the morning

Initiate within 1 year after completion of trastuzumab therapy and take continuously for 1 year

Adjust dose in $40 \mathrm{mg}$ decrements to manage adverse events (minimum dose $120 \mathrm{mg}$ )

How should neratinib be used in special populations?

Patients with renal impairment Mild to moderate impairment: no dosage adjustment required

Severe impairment or on dialysis: not recommended (lack of data)

Patients with hepatic impairment Mild to moderate (Child-Pugh A or B): no dosage adjustment required

Severe impairment (Child-Pugh C): contraindicated

Women Of child-bearing potential: must use highly effective contraceptive measures (may cause foetal harm)

During pregnancy: should not use (lack of data; may cause of foetal harm)

Women who are breastfeeding: use only if the potential benefit to mother outweighs the benefit of breastfeeding the child (lack of data)

Elderly patients No dosage adjustment required; monitor carefully for renal insufficiency and dehydration (age-related risk)

What other special warnings/precautions/monitoring requirements pertain to the use of neratinib?

Diarrhoea Initiate prophylaxis with an antidiarrhoeal agent with the first dose of neratinib, maintain regular dosing of the antidiarrhoeal in the first 1-2 months, and titrate to 1-2 bowel movements/day

Appropriate dosage adjustment of neratinib and dietary changes are required

Hepatotoxicity Monitor ALT, AST and total bilirubin levels at week 1, then monthly for the first 3 months and every 6 weeks thereafter during treatment or as clinically indicated

Monitor liver function tests in patients with grade $\geq 3$ diarrhoea requiring IV fluid treatment, and patients with signs or symptoms of hepatotoxicity

Left ventricular dysfunction $\quad$ Conduct cardiac monitoring in patients with known cardiac risk factors (lack of data in patients with significant cardiac history or left ventricular ejection fraction less than the lower limit of normal)

Skin and subcutaneous tissue disorders Monitor carefully in symptomatic patients

What are the potential clinically relevant interactions between neratinib and other drugs?

PPIs and H2-receptor antagonists

Not recommended

Antacids

Separate dosing of neratinib and antacids by $\geq 3 \mathrm{~h}$

Strong CYP3A4/P-gp inducer

Contraindicated (may decrease systemic exposure to neratinib)

Moderate CYP3A4/P-gp inhibitors

Contraindicated (may increase systemic exposure to neratinib)

Consult local prescribing information for further details

ALT alanine aminotransferase, AST aspartate aminotransferase, CYP cytochrome P450, HER2 human epidermal growth factor receptor 2, $I V$ intravenous, $P$ - $g p$ P-glycoprotein, $P P I$ proton pump inhibitor

for neratinib and placebo was seen at $\approx 3$ months and was maintained throughout the 5-year follow-up period [16]. DFS including ductal carcinoma in situ was also significantly improved with neratinib relative to placebo at 2 and 5 years, but there were no significant differences between the neratinib and placebo groups for distant DFS, time to distant recurrence or cumulative incidence of CNS recurrence at these timepoints (Table 2) [6]. Overall survival data are not yet mature $[15,16]$.

Health-related quality of life (HR-QOL) deteriorated slightly with neratinib during month 1 , as assessed by the difference in adjusted mean change from baseline versus placebo in the Functional Assessment of Cancer TherapyBreast (FACT-B) total scores and EuroQol 5-Dimensions
(EQ-5D) visual analogue scale scores, but this was not considered clinically important [15]. In both treatment groups the two HR-QOL scores recovered towards baseline scores after the first month and the between-group differences became less pronounced [15].

In subgroup analyses at 2 and 5 years, improvements in invasive DFS consistent with those in the intent-to-treat population were seen in almost all subgroups $(n=101-2297)$, regardless of demographic and disease characteristics (HRs $<1 ; p$ interaction at 5 years nonsignificant for all subgroups) $[15,16]$. A prespecified subgroup analysis of invasive DFS at 2 years in patients stratified according to HRc status suggested significant benefit with neratinib relative to placebo in 1631 patients with HRc-positive disease [95 vs. 
Original Protocol (Wyeth, Apr 2009)

Primary objective: compare iDFS with NER

versus PL in women with early-stage HER2

overexpressed/amplified BC following TRA in the extended adjuvant setting

Amendment 3 (Pfizer, Feb 2010)

Patients with stage $2-3 \mathrm{BC}$ at higher risk of

recurrence, i.e. those with node-positive disease and randomized $\leq 1$ year after TRA

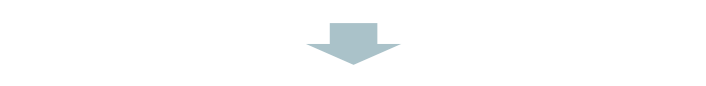

Amendment 9 (Pfizer, Oct 2011)

Stop enrolment of new subjects and limit the

follow-up period to 2 years post-randomization

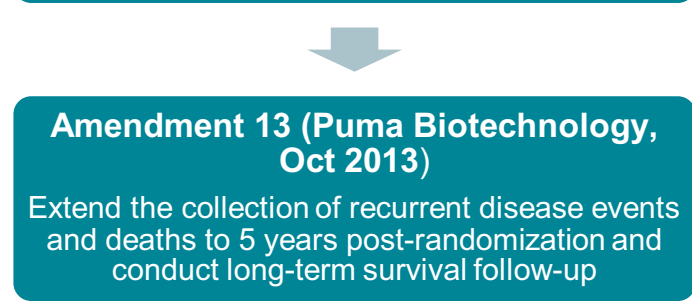

Fig. 2 Key global protocol amendments of the ExteNET study. $B C$ breast cancer, $H E R 2$ human epidermal growth factor receptor 2, $i D F S$ invasive disease-free survival, $N E R$ neratinib, $P L$ placebo, TRA trastuzumab

91\%; hazard ratio (HR) 0.51 ; 95\% CI 0.33-0.77], but not in 1209 patients with HRc-negative disease (92 vs. 92\%; 0.93; 95\% CI 0.60-1.43) [15, 16]. In patients stratified according to the timing of neratinib, those who initiated neratinib $\leq 1$ year of completing trastuzumab adjuvant therapy appeared to have greater treatment benefit (HR 0.65; 95\% CI 0.47-0.89) than those who received neratinib $>1$ year after trastuzumab (HR 0.92; 95\% CI 0.37-2.23), although the results were limited by the small number of patients $(n=2297$ and 532, respectively) $[15,16]$. The significant invasive DFS benefit with neratinib relative to placebo at 2 years was sustained at the 5-year follow-up, both in patients with HRc-positive disease (91 vs. $87 \%$; HR 0.60; 95\% CI $0.43-0.83$ ) and in patients who received treatment $\leq 1$ year after trastuzumab (90 vs. 87\%; HR 0.70; 95\% CI 0.54-0.90) [15, 16].

\subsection{HRc-Positive Patients Treated $\leq 1$ Year After Trastuzumab}

An exploratory analysis showed that the subgroup of patients in ExteNET who had HRc-positive, HER2-positive disease and had received neratinib $\leq 1$ year from completion of trastuzumab $(n=1334)$ derived the greatest benefit with neratinib, as indicated by a $51 \%$ reduction in the risk of invasive disease recurrence or death at 2 years $[6,17]$, with the treatment benefit maintained at the 5 -year followup [16, 17] (Table 2). The Kaplan-Meier curves for invasive DFS for neratinib and placebo recipients separated at $\approx 3$ months and remained separate throughout the 5-year follow-up period [17]. DFS including ductal carcinoma, distant DFS and time to distant recurrence were also significantly improved with neratinib relative to placebo at 2 and 5 years post-randomization (Table 2). Although the cumulative incidence of CNS recurrence was numerically lower with neratinib than with placebo both at 2 and 5 years, the between-group difference did not reach statistical significance (Table 2) [16, 17].

In another exploratory analysis of patients with HRc-positive tumours who started treatment $\leq 1$ year of completing trastuzumab but did not achieve a pathological complete response after neoadjuvant therapy $(n=295)$, the invasive DFS rate at 2 years was $90 \%$ with neratinib versus $85 \%$ with placebo (HR $0.64 ; 0.30-1.29$ ) and at 5 years was 85 versus 78\% (HR 0.60; 95\% CI 0.33-1.07) [17].

\section{What is the Tolerability of Neratinib?}

Neratinib had an acceptable safety profile in the large ExteNET trial in patients with early-stage HER2-positive breast cancer who had completed adjuvant trastuzumab therapy $[15,16]$. The most common any-grade or grade $\geq 3$ treatment-emergent AE with neratinib was diarrhoea (Fig. 3) [15]. Of note, antidiarrhoeal prophylaxis was not specified in the study protocol, instead treatment for diarrhoea was advised once symptoms became apparent. Other grade 3 or 4 AEs each occurred in $<4 \%$ of patients in the neratinib group, with non-gastrointestinal events generally occurring at similar incidences in neratinib and placebo recipients. QT prolongation was reported in 3\% of neratinib and 7\% of placebo recipients and grade $\geq 2$ decreases in LVEF in $1 \%$ of patients in each group. Interstitial lung disease occurred in two patients in the neratinib group and one patient in the placebo group, pulmonary fibrosis in one and two patients, respectively, and pneumonitis in one patient in each group; second cancers (neoplasms benign, malignant and unspecified, including cysts and polyps) occurred in $1 \%$ of patients in each group [15].

Abnormalities of liver function tests, indicating potential cases of hepatotoxicity, occurred almost twice as frequently in the neratinib as in the placebo group (12 vs. 7\%), largely because of elevated ALT ( 9 vs. 3\%), AST (7 vs. 3\%) and blood alkaline phosphatase ( 2 vs. $1 \%$ ) levels; grade 3 or 4 hepatic-associated adverse reactions occurred in $<2$ vs. 
Table 2 Efficacy of oral neratinib $240 \mathrm{mg}$ once daily as extended adjuvant therapy in patients with HER2-positive early stage breast cancer in the ExteNET randomized, double-blind, multicentre study

\begin{tabular}{|c|c|c|c|c|c|c|}
\hline \multirow{2}{*}{$\begin{array}{l}\text { Median } \\
\text { follow-up }\end{array}$} & \multirow{2}{*}{$\begin{array}{l}\text { Treatment } \\
\text { (no. of pts) }\end{array}$} & \multicolumn{5}{|c|}{ Event-free survival rate (\%) [NER vs. PL hazard ratio; 95\% CIs] } \\
\hline & & $\mathrm{iDFS}^{\mathrm{a}}$ & $\mathrm{DFSdc}^{\mathrm{b}}$ & $\mathrm{dDFS}^{\mathrm{c}}$ & $\mathrm{TDR}^{\mathrm{d}}$ & $\mathrm{rCNS}^{\mathrm{e}}$ \\
\hline \multicolumn{7}{|c|}{ Intent-to-treat population } \\
\hline \multirow[t]{2}{*}{2 years $[6]$} & NER (1420) & $94.2[0.66 ; 0.49-0.90]^{* * \mathrm{f}}$ & $94.2[0.61 ; 0.45-0.83]^{* * *}$ & $95.3[0.74 ; 0.52-1.05]$ & $95.5[0.73 ; 0.51-1.04]$ & 0.92 \\
\hline & PL (1420) & $91.9^{f}$ & 91.3 & 94.0 & 94.2 & 1.16 \\
\hline \multirow[t]{2}{*}{5 years $[16]$} & NER (1420) & $90.2[0.73 ; 0.57-0.92]^{* *}$ & $89.7[0.71 ; 0.56-0.89]^{* * *}$ & $91.6[0.78 ; 0.60-1.01]$ & $91.8[0.79 ; 0.60-1.03]$ & 1.3 \\
\hline & PL (1420) & 87.7 & 86.8 & 89.9 & 90.3 & 1.8 \\
\hline \multicolumn{7}{|c|}{ HRc + patients who are $\leq 1 \mathrm{y}$ from completion of TRA } \\
\hline \multirow[t]{2}{*}{2 years $[6,17]$} & NER (670) & $95.3[0.49 ; 0.30-0.78]^{* * *}$ & $95.3[0.45 ; 0.28-0.71]^{* * *}$ & $96.1[0.53 ; 0.31-0.88]^{*}$ & $96.3[0.53 ; 0.30-0.89]^{*}$ & 0.34 \\
\hline & PL (664) & 90.8 & 90.0 & 92.9 & 93.3 & 1.01 \\
\hline \multirow[t]{2}{*}{5 years $[17]$} & NER (670) & $90.8[0.58 ; 0.41-0.82]^{* * *}$ & NR $[0.55 ; 0.39-0.77] * * *$ & $92.4[0.57 ; 0.39-0.83]^{* * *}$ & NR $[0.58 ; 0.39-0.85]^{* * *}$ & 0.69 \\
\hline & PL (664) & 85.7 & NR & 87.7 & NR & 2.09 \\
\hline
\end{tabular}

$B C$ breast cancer, $d D F S$ distant disease-free survival, $D F S d c$ disease-free survival including ductal carcinoma in situ, $H R c$ hormone receptor, $i D F S$ invasive disease-free survival, $N E R$ neratinib, $N R$ not reported, $P L$ placebo, $r C N S$ central nervous system recurrence, $T D R$ time to distant recurrence, TRA trastuzumab

$* p<0.05, * * p<0.01, * * * p \leq 0.005$

${ }^{a}$ Invasive ipsilateral tumour recurrence, invasive contralateral BC, local or regional invasive recurrence, distant recurrence, or death from any cause

${ }^{\mathrm{b}}$ Time from randomization to the first occurrence of a DFS event or ductal carcinoma in situ event

${ }^{\mathrm{c}}$ Time from randomization to the first occurrence of distant recurrence or death from any cause

${ }^{\mathrm{d}}$ Time between randomization and the date of the first distant recurrence or death from BC

${ }^{\mathrm{e}}$ Cumulative incidence; defined as time from randomization to rCNS as first distant recurrence

${ }^{\mathrm{f}}$ Primary endpoint

$<1 \%$ of patients in the neratinib and placebo groups [6]. Thus, monitoring of liver function tests is recommended for patients receiving neratinib therapy (Table 1) [6].

Serious treatment-emergent AEs occurred in $7 \%$ of neratinib and $6 \%$ of placebo recipients, with diarrhoea [ 22 vs. 1 patient(s)], vomiting [12 vs. 1 patient(s)] and dehydration [9 vs. 1 patient(s)] being reported most commonly [15]. Treatment-emergent AEs resulted in dose reductions in $31 \%$ and $2 \%$ of patients in the neratinib and placebo groups, and treatment discontinuations in $28 \%$ and $5 \%$ of patients [16]. At the time of the 2-year analysis, four deaths in the neratinib group and three deaths in the placebo group unrelated to disease progression were reported after treatment discontinuation, none of which were considered to be drug related [15]. At the time of the 5-year analysis, 121 deaths had occurred in the neratinib and placebo groups combined (data are masked), of which 102 deaths were because of disease progression and 19 deaths were due to other reasons [16]. A review of unsolicited serious AEs occurring $>28$ days after the last dose of the study drug reported to a safety database showed no evidence of increased long-term toxicity with neratinib relative to placebo, particularly symptomatic cardiac toxicity or second primary malignancies [16].

The safety profile of neratinib in the subgroup of patients with HRc-positive, HER2-positive breast cancer who initiated neratinib $\leq 1$ year from completion of trastuzumab was generally similar to that in the overall safety population [17]. The most common grade $\geq 3$ treatment-emergent AEs with neratinib included diarrhoea $(39 \%$ vs. $1 \%$ with placebo), fatigue $(2 \%$ vs. $<1 \%)$ and nausea $(1 \%$ vs. $<1 \%)$. Treatment-emergent AEs resulted in dose reductions in $31 \%$ of neratinib and $2 \%$ of placebo recipients, dose holds in $42 \%$ and $11 \%$, and hospitalizations in $6 \%$ and $5 \%$ of patients, respectively [17].

\subsection{Diarrhoea}

Diarrhoea was the main toxicity associated with neratinib in ExteNET (Fig. 3), leading to dose reductions in 26\% (vs. $1 \%$ in placebo recipients), hospital admissions in $1 \%$ (vs. $<1 \%$ ) and discontinuation of therapy in $17 \%$ (vs. $<1 \%$; after median 20 vs. 241 days) of neratinib recipients in the absence of antidiarrheal prophylaxis [15]. Most grade 3 diarrhoea events with neratinib were reported during the first month (cycle 1) of treatment, with a median time to onset of 8 days, median cumulative duration of 5 days/patient and median duration of 2 days/event [15], supporting the use of preventive management with antidiarrheal prophylaxis early during neratinib treatment. In pooled data from 1660 patients who received neratinib monotherapy without loperamide prophylaxis, 95\% of patients had at least one episode of diarrhoea, and diarrhoea was the most common 


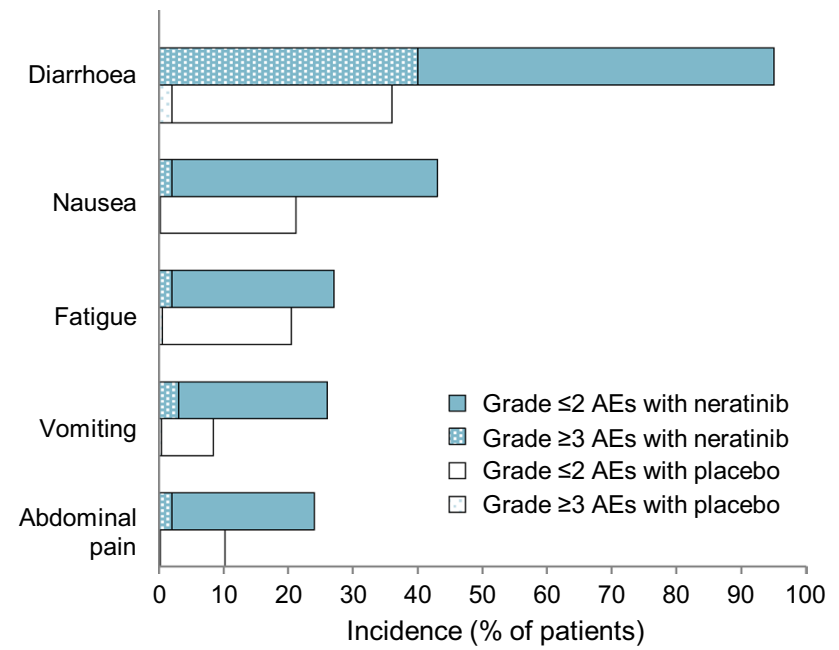

Fig. 3 Any-grade adverse events occurring at an incidence of $\geq 20 \%$ with neratinib $(n=1408)$ and placebo $(n=1408)$ as extended adjuvant therapy in the ExteNET study. AEs adverse events

reason for treatment discontinuation (14\%); $25 \%$ of patients required dose reductions because of diarrhoea [6]. Grade 3 diarrhoea occurred in $38 \%$ of neratinib recipients in this analysis, and grade 4 diarrhoea in $0.2 \%$ of patients; $2 \%$ of patients required hospitalization as a result of diarrhoea [6].

To manage neratinib-associated diarrhoea, it is recommended that antidiarrhoeal prophylaxis be initiated with the first dose of neratinib and continued for the first 1-2 months of therapy [6]. Dosage adjustment or temporary discontinuation of neratinib may also be required to manage diarrhoea (Table 1) [6].

An international, open-label, sequential-cohort, phase 2 CONTROL (PUMA-NER-6201) study is assessing the effects of loperamide prophylaxis alone or with add-on budesonide (a locally acting corticosteroid used for inflammatory gastrointestinal conditions) or colestipol (a bile acid sequestrant) on neratinib-associated diarrhoea [18]. The study enrolled adults who had HER2-positive stage $1-3 c$ breast cancer and had completed trastuzumab-based adjuvant therapy or had discontinued treatment as a result of AEs, with the last dose of trastuzumab $>2$ weeks and $\leq 1$ year prior to enrolment. All patients received neratinib $240 \mathrm{mg} /$ day in 28 -day cycles for 1 year ( 13 cycles); in addition, patients received loperamide prophylaxis for 1 or 2 cycles and then as needed ( $4 \mathrm{mg}$ initial dose, then 6-12 mg/ day; $n=137)$, loperamide with add-on budesonide (9 $\mathrm{mg}$ once daily for 1 cycle; $n=64$ ) or loperamide with add-on colestipol ( $2 \mathrm{~g}$ twice daily for 1 cycle; $n=120)$. At the time of an interim analysis (cut-off date November 2017), the median duration of neratinib therapy was $11.5,11.9$ and 3.7 months in the loperamide, budesonide and colestipol cohorts, respectively. The primary endpoint was the incidence of grade $\geq 3$ diarrhoea [18].
Concomitant antidiarrhoeal treatment appeared to reduce the duration, incidence and severity of diarrhoea in patients receiving neratinib in CONTROL [18]. In terms of grade $\geq 3$ diarrhoea, add-on colestipol was associated with the greatest reduction in the incidence relative to the other loperamide cohorts or the ExteNET trial as the historical control $(11,27$ and $31 \%$ in the colestipol, budesonide and loperamide cohorts vs. $40 \%$ in ExteNET). Grade 1 or 2 diarrhoea occurred in 49,59 and $56 \%$ of patients in the loperamide, loperamide + budesonide and loperamide + colestipol cohorts in CONTROL compared with 55\% of patients in ExteNET; no diarrhoea was reported in 20, 14 and 33\% of patients in the respective cohorts compared with $5 \%$ of patients in ExteNET [18].

The median cumulative duration of any-grade diarrhoea in all three loperamide cohorts (loperamide, loperamide + budesonide and loperamide + colestipol) in CONTROL appeared to be shorter (any grade: 14, 24 and 16 vs. 59 days in ExteNET) and the proportions of patients requiring dose reductions $(7,3,4$ vs. $26 \%)$ or dose holds $(15,19$ and 9 vs. $34 \%)$ because of diarrhoea appeared to be lower than those in the ExteNET trial [18]. The CONTROL study cohorts also had reduced incidences and severity of diarrhoea relative to ExteNET during the course of neratinib therapy. In ExteNET, grade 2 and 3 diarrhoea had the greatest incidence during cycle 1 (month 1) and was still occurring during months $2-12$ (chronic profile). In comparison, in the CONTROL study cohorts, the incidence of grade 2 or 3 diarrhoea was reduced during cycle 1 (month 1 ) and in months 2-12[18].

\section{What Conclusions can be Made Regarding the Clinical Use of Neratinib?}

In the EU, the standard treatment for early-stage breast cancer in most cases is surgical resection with or without radiation therapy, and/or systemic adjuvant therapy [19]. The choice of systemic therapy is based on the surrogate intrinsic phenotype determined by HRc and HER 2 status and assessment of the proliferation marker Ki67 [19]. For patients with HER2-positive disease, the anti-HER2 monoclonal antibody trastuzumab has become the mainstay of treatment, with current guidelines recommending trastuzumab therapy for 1 year in combination with chemotherapy [1]. For patients at high risk of recurrence, the anti-HER2 monoclonal antibody pertuzumab has been approved as neoadjuvant and adjuvant therapy in combination with trastuzumab and chemotherapy [20]. In addition, patients with HRc-positive disease are administered endocrine therapy for at least 5 years [1].

Despite the improved clinical outcomes with trastuzumab, the issue of high recurrence rates remains, which has spurred the development of new therapies. One such agent is the 
irreversible, pan-HER tyrosine kinase inhibitor neratinib, which has been developed as extended adjuvant therapy to reduce the risk of recurrence in patients with HER2-positive early-stage breast cancer. Treatment with neratinib for 12 months significantly improved the primary endpoint of invasive DFS relative to placebo at both 2 and 5 years in the pivotal ExteNET study in women with early-stage HER2-positive breast cancer who had completed adjuvant trastuzumab (Sect. 4.1). The secondary endpoint of DFS including ductal carcinoma in situ was also improved and HR-QOL was not adversely affected by neratinib relative to placebo (Sect. 4.1). It has been suggested that the improved clinical outcomes with neratinib as adjuvant therapy could be due to irreversible kinase inhibition through the binding of neratinib to a cysteine residue in the ATP-binding pocket, resulting in more effective blockade of HER2 with neratinib than with trastuzumab [15].

Notably, subgroup analyses of ExteNET suggested that patients with HRc-positive disease derived greater benefit with neratinib than patient with HRc-negative disease, and patients who initiated neratinib $\leq 1$ year of completing trastuzumab had better outcomes than those who started treatment 1-2 years after trastuzumab (Sect. 4.1). Subsequent analyses showed that patients with HRc-positive disease who received treatment $\leq 1$ year after trastuzumab derived the greatest treatment benefit, with significant improvement in invasive DFS and other efficacy outcomes, apart from the cumulative incidence of CNS recurrence, for which event numbers remained small (Sect. 4.2).

It has been suggested that the improved efficacy in patients with HRc-positive disease may be because of dual inhibition of ER-HER2 crosstalk by neratinib and endocrine therapy (which was administered to the majority of patients in this subgroup). Another explanation may relate to the timing of adjuvant neratinib therapy and the different clinical course of HRc-negative and HRc-positive subgroups in terms of the risk of recurrence [13]. HRc-negative patients are at highest risk of recurrence in the first few years after completion of adjuvant trastuzumab, while HRc-positive patients are at a long-term continuous risk of recurrence [21]. In patients with HER2-positive breast cancer who were disease-free after completion of prior adjuvant chemotherapy and who were not treated with antiHER 2 therapy (placebo arm of the TEACH trial), the mean risk of recurrence in years $1-5$ was $9 \%$ per year in patients with HRc-negative disease compared with $5 \%$ per year for patients with HRc-positive disease $(p=0.002)$ [22]. The high risk of recurrence in patients with HRc-negative disease declined sharply thereafter, such that there was no significant difference between the HRc-positive and -negative subgroups in years 6-10 (mean risk of 4\% per year in both groups) [22]. This suggests that there is a longer window of opportunity to provide benefit with extended adjuvant therapy in patients with HRc-positive disease [13].
Neratinib had a generally acceptable safety profile in ExteNET, with the most common any-grade or grade $\geq 3$ treatment-emergent AE being diarrhoea, which is considered a drug class effect [16]. The highest incidence of diarrhoea with neratinib was in cycle 1 of treatment, which may explain the decrease in HR-QOL scores during this period [15]. The drug of choice for the management of diarrhoea associated with tyrosine kinase inhibitors is the opiod loperamide, as it has local activity in the gut and is minimally absorbed and thus not associated with systemic effects [23]. Indeed, intensive loperamide prophylaxis at the start of neratinib therapy in phase 1 and phase $1 / 2$ studies was associated with improved tolerability of neratinib, as indicated by fewer grade 3 diarrhoea events in patients receiving intensive loperamide prophylaxis (incidence 0-17\%) compared with no or suboptimal loperamide prophylaxis (incidence 30-53\%) [24]. In ExteNET, treatment with loperamide was initiated only after the development of symptoms, and grade 3 diarrhoea was reported in $40 \%$ of neratinib recipients (Fig. 3). Because of the risk of severe diarrhoea with neratinib, prophylactic antidiarrhoeal treatment is recommended, and should be initiated with the first dose of neratinib and continued for 1-2 cycles [6]. Interim results from the ongoing, phase 2 CONTROL study suggest that the addition of colestipol to loperamide may further reduce the incidence and duration of grade $\geq 3$ diarrhoea (Sect. 5.1). Neratinib was not associated with long-term toxicity in a safety database review, particularly symptomatic cardiac toxicity or second primary malignancies (Sect. 5).

The ExteNET study protocol underwent several amendments during the course of the study as three different sponsors assumed responsibility (Fig. 2), including cessation of enrolment and shortening of follow-up to 2 years, and subsequent restoration of follow-up to 5 years $[15,16]$. As a result, reconsent for long term follow-up was required for most patients and, because of missing data, there is some uncertainty regarding the magnitude of effect at 5 years $[13,16]$. However, the effect size observed at the 5-year follow-up suggests a clinically significant benefit in HRc-positive patients who received neratinib $\leq 1$ from completion of trastuzumab [13].

Given its efficacy and manageable safety, neratinib was recently approved as extended adjuvant treatment of patients with early-stage HRc-positive, HER2-positive breast cancer who are $<1$ year from the completion of trastuzumab therapy [6]. Although the exact position of neratinib in the treatment algorithm for early-stage breast cancer (for example in relation to adjuvant pertuzumab therapy) remains to be determined [25], neratinib provides a valuable treatment option to reduce the risk of recurrence in these patients. The recently updated ESMO patient guide also includes neratinib as an extended adjuvant therapy for some patients following the completion of trastuzumab therapy [26]. 
Acknowledgements The manuscript was reviewed by: J. Jeong, Department of Surgery, Gangnam Severance Hospital, Yonsei University College of Medicine, Seoul, Republic of Korea; F. Montemurro, Multidiscliplinary Oncology Outpatient Clinic, Candiolo Cancer Institute, FPO-IRCCS, Cadiolo, Italy; F. Muggia, NYU Cancer Institute, Kaplan Cancer Center, New York University Medical Center, New York, NY, USA. During the peer review process, the manufacturer of neratinib was offered an opportunity to review this article. Changes resulting from comments received were made on the basis of scientific and editorial merit.

\section{Compliance with Ethical Standards}

Funding The preparation of this review was not supported by any external funding.

Conflicts of interest S. Dhillon is a salaried employee of Adis/Springer, is responsible for the article content and declares no relevant conflicts of interest.

Open Access This article is distributed under the terms of the Creative Commons Attribution-NonCommercial 4.0 International License (http://creativecommons.org/licenses/by-nc/4.0/), which permits any noncommercial use, duplication, adaptation, distribution and reproduction in any medium or format, as long as you give appropriate credit to the original author(s) and the source, provide a link to the Creative Commons license and indicate if changes were made.

\section{References}

1. Mitri Z, Constantine T, O'Regan R. The HER2 receptor in breast cancer: pathophysiology, clinical use, and new advances in therapy. Chemother Res Pract. 2012;2012:743193.

2. Wieduwilt MJ, Moasser MM. The epidermal growth factor receptor family: biology driving targeted therapeutics. Cell Mol Life Sci. 2008;65(10):1566-84.

3. Lambertini M, Ponde NF, Solinas C, et al. Adjuvant trastuzumab: a 10-year overview of its benefit. Expert Rev Anticancer Ther. 2017;17(1):61-74.

4. Kourie HR, El Rassy E, Clatot F, et al. Emerging treatments for HER2-positive early-stage breast cancer: focus on neratinib. Onco Targets Ther. 2017;10:3363-72.

5. Puma Biotechnology. Nerlynx (neratinib): US prescribing Information. 2017. https://www.accessdata.fda.gov/drugsatfda_docs/ label/2017/208051s000lbl.pdf. Accessed 4 Dec 2018.

6. European Medicines Agency. Nerlynx (neratinib): summary of product characteristics. 2018. https://www.ema.europa.eu/docum ents/product-information/nerlynx-epar-product-information en.pdf. Accessed 4 Dec 2018.

7. Puma Biotechnology I. European Commission grants Marketing Authorisation for Puma Biotechnology's NERLYNX ${ }^{\circledR}$ (neratinib) for extended adjuvant treatment of hormone receptor positive HER2-positive early stage breast cancer [media release]. 4 Sept 2018. http://www.pumabiotechnology.com/pr20180904.html.

8. Rabindran SK, Discafani CM, Rosfjord EC, et al. Antitumor activity of HKI-272, an orally active, irreversible inhibitor of the HER-2 tyrosine kinase. Cancer Res. 2004;64(11):3958-65.

9. Canonici A, Gijsen M, Mullooly M, et al. Neratinib overcomes trastuzumab resistance in HER2 amplified breast cancer. Oncotarget. 2013;4(10):1592-605.

10. Luque-Cabal M, Garcia-Teijido P, Fernandez-Perez Y, et al. Mechanisms behind the resistance to trastuzumab in HER2-amplified breast cancer and strategies to overcome it. Clin Med Insights Oncol. 2016;10(Suppl 1):21-30.

11. Feldinger K, Kong A. Profile of neratinib and its potential in the treatment of breast cancer. Breast Cancer (Dove Med Press). 2015; 7:147-62.

12. Giuliano M, Trivedi MV, Schiff R. Bidirectional crosstalk between the estrogen receptor and human epidermal growth factor receptor 2 signaling pathways in breast cancer: molecular basis and clinical implications. Breast Care. 2013;8(4):256-62.

13. European Medicines Agency. Nerlynx (neratinib): assessment report. 2018. https://www.ema.europa.eu/documents/assessment -report/nerlynx-epar-public-assessment-report_en.pdf. Accessed 4 Dec 2018.

14. Sudhan DR, Schwarz LJ, Guerrero-Zotano A, et al. Extended adjuvant therapy with neratinib plus fulvestrant blocks ER/HER2 crosstalk and maintains completerResponses of ER(+)/HER2(+) breast cancers: implications to the ExteNET trial. Clin Cancer Res. 2018

15. Chan A, Delaloge S, Holmes FA, et al. Neratinib after trastuzumab-based adjuvant therapy in patients with HER2-positive breast cancer (ExteNET): a multicentre, randomised, double-blind, placebo-controlled, phase 3 trial. Lancet Oncol. 2016;17(3):367-77.

16. Martin M, Holmes FA, Ejlertsen B, et al. Neratinib after trastuzumab-based adjuvant therapy in HER2-positive breast cancer (ExteNET): 5-year analysis of a randomised, double-blind, placebo-controlled, phase 3 trial. Lancet Oncol. 2017;18(12):1688-700.

17. Gnant M, Martin M, Holmes FA, et al. Efficacy of neratinib in hormone receptor-positive patients who initiated treatment within 1 year of completing trastuzumab-based adjuvant therapy in HER2 + early stage breast cancer: subgroup analyses from the phase III ExteNET trial [abstract no. 603 plus poster]. In: San Antonio Breast Cancer Symposium 2018. http://www.pumabiotechnology.com/docs/12061 8_Puma_ExteNET_Poster_at_SABCS_2018.pdf

18. Hurvitz S, Chan A, Iannotti N, et al. Effects of adding budesonide or colestipol to loperamide prophylaxis on neratinib-associated diarrhea in patients with HER2 + early-stage breast cancer: the CONTROL trial [abstract no. P3-14-01 plus poster]. Cancer Res. 2017;78(4 Suppl). http://www.pumabiotechnology.com/docs/ P3-14-01120517PBYIatSABCSCONTROLPoster.pdf

19. Senkus E, Kyriakides S, Ohno S, et al. Primary breast cancer: ESMO Clinical Practice Guidelines for diagnosis, treatment and follow-up. Ann Oncol. 2015;26(Suppl 5):v8-30.

20. European Medicines Agency. Perjeta (pertuzumab): summary of product characteristics. 2018. https://www.ema.europa.eu/docum ents/product-information/perjeta-epar-product-information_en-0. pdf. Accessed 4 Dec 2018.

21. Untch M, Gelber RD, Jackisch C, et al. Estimating the magnitude of trastuzumab effects within patient subgroups in the HERA trial. Ann Oncol. 2008;19(6):1090-6.

22. Strasser-Weippl K, Horick N, Smith IE, et al. Long-term hazard of recurrence in HER2 + breast cancer patients untreated with antiHER2 therapy. Breast Cancer Res. 2015;17:56.

23. Bossi P, Antonuzzo A, Cherny NI, et al. Diarrhoea in adult cancer patients: ESMO Clinical Practice Guidelines. Ann Oncol. 2018;29(Suppl 4):iv126-42.

24. Ustaris F, Saura C, Palma JD, et al. Effective management and prevention of neratinib-induced diarrhea. Am J Hematol Oncol. 2015;11(11):13-22.

25. Chavez-MacGregor M, Mittendorf EA. Extended adjuvant therapy in patients with HER2-positive breast cancer: some answers, even more questions. Lancet Oncol. 2017;18(12):1568-9.

26. ESMO. Breast Cancer: an ESMO guide for patients. 2018. https ://www.esmo.org/content/download/6593/114959/file/EN-Breas t-Cancer-Guide-for-Patients.pdf. Accessed 4 Dec 2018. 